\title{
A Rare Case Report of Primary Pure Pleomorphic Liposarcoma of Breast with Cytological and Histopathological Findings
}

\author{
Mukherjee $A^{1}$, Nath $J^{1}$, Dey $D^{1}$, Chakravorty $S^{1}$, Sinha $S^{1}$ and Chatterjee $T^{*_{2}}$ \\ ${ }^{1}$ Suraksha Diagnostic Centre, Salt Lake, Kolkata, West Bengal, India \\ ${ }^{2}$ Suraksha Genomics (R\&D Division of Suraksha Diagnostic), Salt Lake, Kolkata, West Bengal, India
}

*Corresponding author: Chatterjee T, Suraksha Genomics (R\&D Division of Suraksha Diagnostic), Salt Lake, Kolkata, West Bengal, India, Tel: +91-98313252580, E-mail: ctridip@gmail.com, tridip.academic@gmail.com

Citation: Mukherjee A, Nath J, Dey D, Chakravorty S, Sinha S, et al. (2017) A Rare Case Report of Primary Pure Pleomorphic Liposarcoma of Breast with Cytological and Histopathological Findings. J Cancer Sci Clin Oncol 4(1): 102. doi: 10.15744/2394-6520.4.102

Received Date: November 05, 2016 Accepted Date: January 20, 2017 Published Date: January 30, 2017

\begin{abstract}
Sarcomas of breast are rare tumors. Primary mammary liposarcomas are rarer still. Peak incidence of breast liposarcomas in adults occur between 4th and 6th decades. We report a case of primary breast pleomorphic liposarcoma in a 70 years old female patient. FNAC of the tumor was non-specific. However, histopathological along with immunohistichemical studies clinched the diagnosis.

Keywords: Breast sarcoma; Liposarcoma; Pleomorphic
\end{abstract}

\section{Introduction}

Breast sarcomas constitute less than $1 \%$ of all the malignant tumors of the breast and liposarcoma accounts for $0.3 \%$ of all the mammary sarcomas [1-3]. Despite being the second most common (16-18\%) soft tissue sarcoma, liposarcomas rarely present as malignancy within the breast. They can arise in cystosarcoma, or may arise independently in mammary adipose tissue [4]. Neuman in 1862 first reported about breast sarcoma. In 1986, Austin and Dupruee published the largest series of breast liposarcomas, including 20 cases [3]. Liposarcomas are thus the rarest of malignant breast tumors, comprising $0.003 \%$ of breast tumors [1]. We are here reporting a case of primary pleomorphic liposarcoma of the breast in an elderly female patient.

\section{Case Report}

A 70 year old female presented with a lump in the left breast, which she has observed since last 2 years. She noted a sudden and rapid increase in its size in last 15 days period. On physical examination, a well circumscribed lump of around $5 \mathrm{~cm}$ in diameter was felt in the lower outer quadrant of the left breast, not adherent to overlying skin or underlying muscle. There was no nipple discharge or palpable axillary lymph nodes. Mammography revealed a high density mass with irregular margins. Patient was referred to our diagnostic facility for fine needle aspiration cytology.

Cytosmears obtained were stained with MGG and PAP. The initial impression was high grade neoplasm with tumour giant cells (Figure 1 and 2).

Subsequently, the patient underwent modified radical mastectomy and the specimen was sent to surgical pathology department for histopathological evaluation. Sections showed a tumor composed predominantly of adipocytes showing a wide variation in size. Numerous lipoblasts were noted. The cells showed marked nuclear pleomorphism. Bizzare tumour giant cells, brisk mitotic activity including atypical mitotic figures were present (Figure 3 and 4). These histomorphological features are consistent with a malignant pleomorphic lesion with the following differential diagnosis:

1. Malignant phyllodes with liposarcomatous differentiation

2. Metaplastic carcinoma of breast and

3. Pleomorphic liposarcoma.

Immunohistochemical study was carried out. The primary panel of antibodies showed positivity for Vimentin and negativity for Cytokeratin excluding any carcinomatous component and confirmed sarcomatous nature of the lesion. The second series 
of antibodies (S-100, desmin, SMA, CD68, CD34, CD117, HMB45) was selected for further characterisation of the sarcoma. Negativity of the primary tumor cells to all these markers and positivity to MDM2, confirmed the diagnosis to be a primary pleomorphic liposarcoma of the breast (Figure 5).

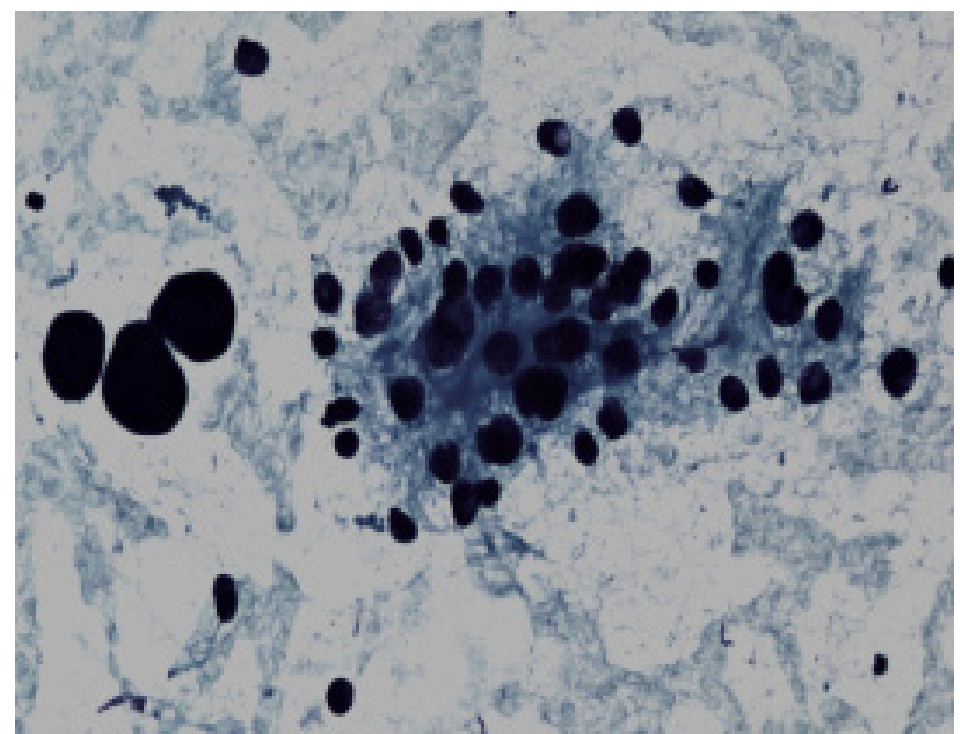

Figure 1: PAP stained FNAC smear (400x): Smear showing cell clusters demonstrating nuclear pleomorphism and bizarre tumor giant cells

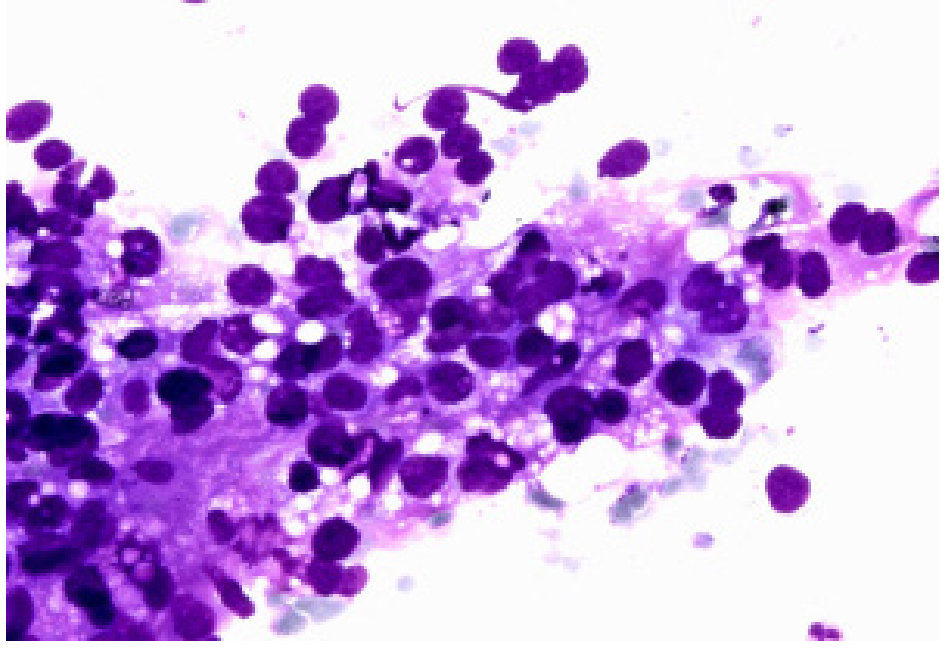

Figure 2: MGG stained FNAC smear (400 X): Cell clusters with nuclear hyperchromasia, irregular nuclear margin and inconspicuous nucleoli

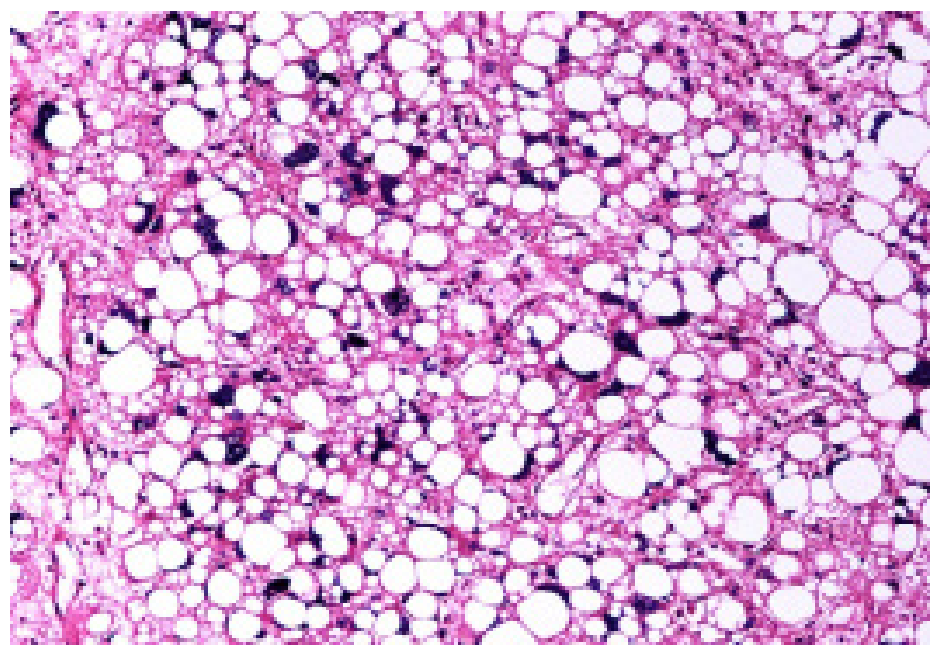

Figure 3: Hematoxylin and eosin: (400x) Presence of bizzare tumour giant cells, numerous lipoblasts with evidence of significant nuclear pleomorphism 


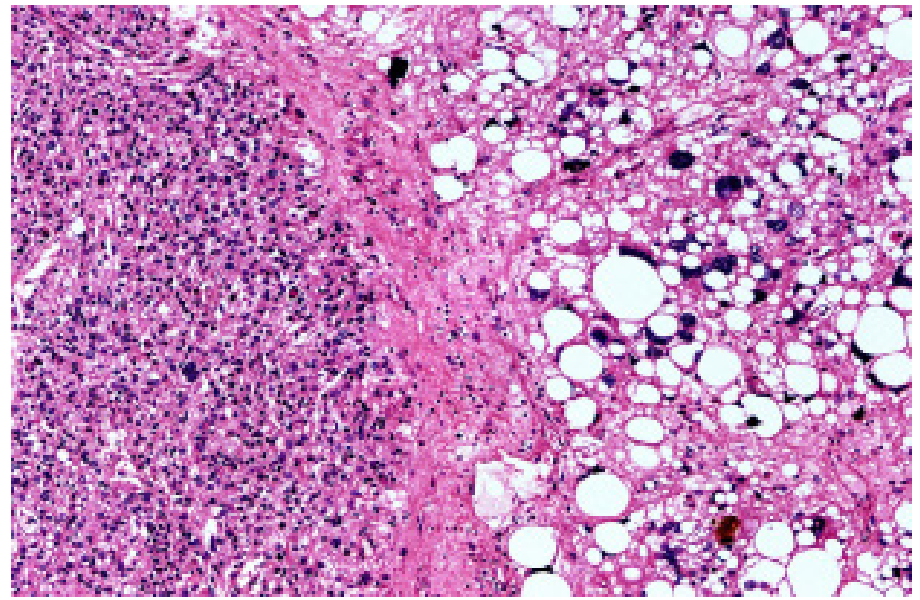

Figure 4: Hematoxylin and eosin: (400x) Tumor composed predominantly of adipocytes on the right showing marked nuclear pleomorphism with brisk mitotic activity and tumor giant cells. Cellular areas of more undifferentiated malignant cells seen on the left

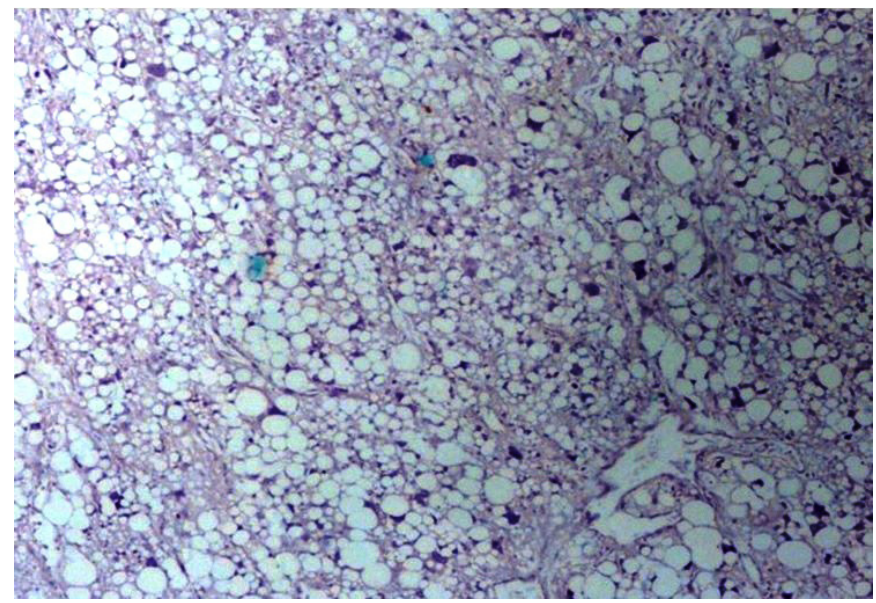

Figure 5: S-100 immunohistochemistry (400x)

\section{Discussion}

Primary sarcomas of breast are rare malignant tumors arising from mesenchymal elements of mammary tissue and lack neoplastic epithelial component. Liposarcomas are the second most common soft tissue sarcomas in adults following malignant fibrous hitiocytoma [4]. Pleomorphic sarcomas are the rarest subtype of liposarcomas and are discriminated from other high grade sarcomas by the presence of pleomorphic lipoblasts. In breast, pleomorphic sarcomas arise either directly from interlobular stromal tissue or as a component of cystosarcoma phyllodes [1]. The age of presentation ranges between 19 to 76 years with a median age of 47 years [1,2]. These tumors generally present as slow growing painful breast masses of variable duration [1]. Breast liposarcomas are generally unilateral at presentation $[1,2]$.

Liposarcoma of breast is a rare condition. Fine needle aspiration cytology can be sufficient for the diagnosis if adequate material is obtained [3]. When a sarcomatous appearing tumor is identified in the breast, it is more likely to be a metaplastic carcinoma or malignant phyllodes rather than a primary sarcoma. Determination of the histological type and differentiation is essential for prognosis and surgical planning of liposarcomas. Pleomorphic liposarcomas are very aggressive high-grade sarcomas. They show a high degree of cellular pleomorphism, including bizzare giant cells [4]. Surgery with local control of the disease is the mainstay of treatment $[1,3,4]$.

\section{References}

1. Mardi K, Gupta N (2011) Primary pleomorphic liposarcoma of breast: A rare case report. Indian J Pathol \& Microbiol 54: 124-6.

2. Üzüm N, Celasin H, Ataoglu O, Kocak S (2010) Pleomorphic Liposarcoma Of The Breast Misdiagnosed As Carcinoma In A Trucut Biopsy. J Breast Heal 2: 87- 90.

3. Akan A, Eryavuz Y, Akarsu C, Bademci R, Filiz F, Sayllgan T (2012) Primary Liposarcoma Of The Breast. J Breast Heal: 8: 89- 91.

4. Whitsell T, Marcovis K, Ruhs S, Andres M, Beck S (2011) High-grade pleomorphic liposarcoma of the breast. Grand Rounds 2011 e- MED Ltd 11: 87-91. 


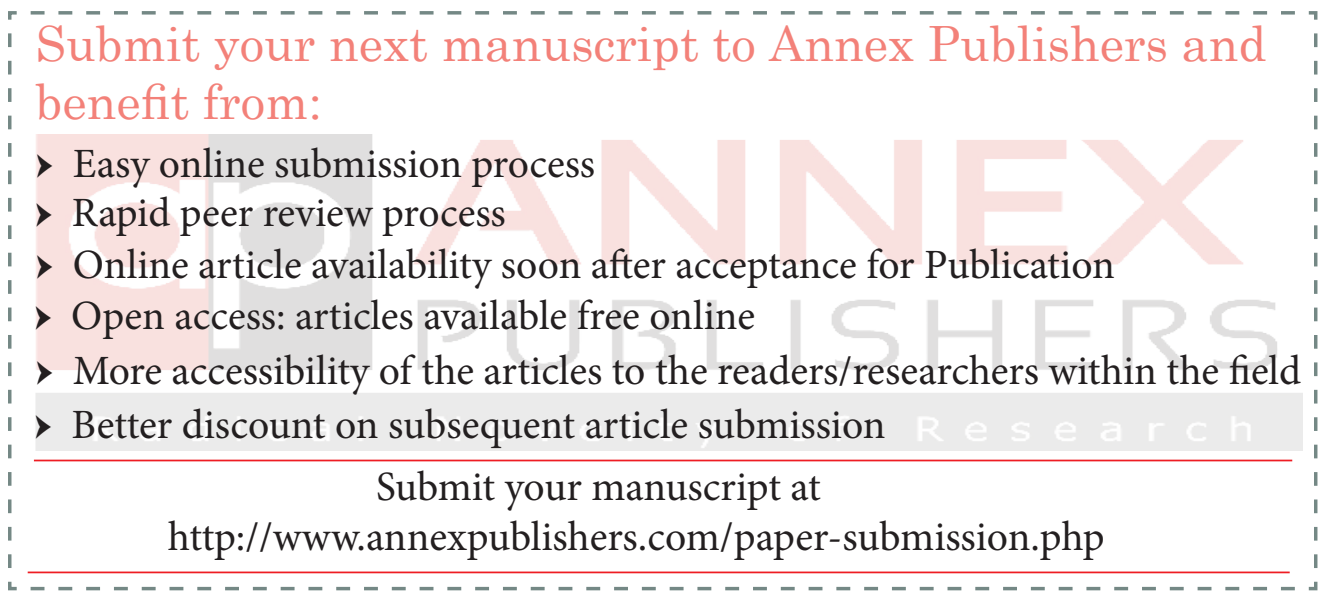

